\title{
Emerging role of brain metastases in the prognosis of breast cancer patients
}

\author{
Amanda Hambrecht ${ }^{1,2}$ \\ Rahul Jandial ${ }^{2}$ \\ Josh Neman ${ }^{2}$ \\ 'Department of Biology, University \\ of Southern California; ${ }^{2}$ Department \\ of Neurosurgery, Beckman Research \\ Institute, City of Hope National \\ Cancer Center, CA, USA
}

This article was published in the following Dove Press journal:

Breast Cancer:Targets and Therapy

9 August 201I

Number of times this article has been viewed

\begin{abstract}
Cancer starts with one rogue cell. Through mutations and genomic alterations, the cell acquires specific and stem cell-like characteristics necessary for invasion of a distant organ and ultimately metastasis. Metastatic brain cancer is a particularly formidable disease because of its poor prognosis and the highly resistant nature of the tumor to chemotherapy. Although several types of primary tumors have a tendency to metastasize to the brain, the incidence of brain metastases has increased dramatically in some subsets of breast cancer patients. Several conventional treatments are available, but success is limited and often short-lived. Given that no standard treatment options exist, there is a significant need to investigate the biology of these clinically recalcitrant tumors.
\end{abstract}

Keywords: metastasis, breast cancer, blood-brain barrier, epithelial-mesenchymal transition, mesenchymal-epithelial transition

\section{Introduction}

The brain has increasingly become the site of untreatable relapse in breast cancer. An estimated 40,000 women die each year from breast cancer, the vast majority due to complications from metastases. ${ }^{1}$ Autopsy studies have shown that up to $25 \%$ of patients who die from cancer develop brain metastases. ${ }^{2}$ The top five primary tumors that lead to brain metastasis are colorectal, renal, melanoma, lung, and breast cancer. Brain metastases have a five-year cumulative incidence rate in $16 \%$ of lung cancer patients, $7 \%$ of breast cancer patients, and $5 \%$ of colon cancer patients. ${ }^{3}$ The incidence rate in melanoma patients has been reported to be as high as $55 \% .{ }^{4}$ However, each type of cancer has a variable period of dormancy prior to relapse in the brain. ${ }^{5}$ The median survival of untreated patients is 1-2 months. Once diagnosed with a brain metastasis, patients have a dismal $20 \%$ probability of surviving more than one year. ${ }^{6,7}$ Brain metastases are highly resistant to chemotherapy and their poor prognosis is primarily due to this chemoresistance. Treatments such as chemotherapy in conjunction with surgery and radiation therapy can only extend patient survival to 4-6 months. Given the emerging role of brain metastases in the prognosis of breast cancer patients, more research studies need to be conducted in order to create therapies and trials for eligible patients with brain metastases as well as to provide a better understanding of the disease.

\section{Epithelial-mesenchymal transition}

During embryonic development, mesenchymal cells form from a primitive epithelium and acquire a morphology necessary for migration in an extracellular environment. 
An epithelial-mesenchymal transition is essential to this process. It is characterized by the conversion of polarized epithelial cells into motile cells. Epithelial cells shed their differentiated characteristics, such as cell adhesion, polar and apical-basal polarity, and lack of motility, and acquire mesenchymal features, including motility, invasiveness, and a heightened response to apoptosis. ${ }^{8}$ The same regulatory mechanisms that convert epithelial cells to migratory mesenchymal cells, which are crucial to the formation of organs during embryonic development, become abnormally activated in cancer and contribute to invasion and metastasis. An essential difference between the embryonic and tumorigenic process is that the latter involves genetically abnormal cells that progressively lose their responsiveness to normal growthregulatory signals. The tumorigenic process generates cancer cells with stem cell-like characteristics. ${ }^{8}$ These cells possess the ability to evolve, which is derived from the genetic and epigenetic instability inherent in most neoplastic cell types. This instability generates distinct subpopulations of cancer cells within larger tumors, constituting a source of phenotypic heterogeneity. ${ }^{8}$ The initiation of an epithelial-mesenchymal transition can occur from several extracellular signals and significant crosstalk, in the form of multiple positive feedback loops, among downstream intracellular signaling pathways and transcription factors. ${ }^{8-10}$ This network of interactions leads to increased stability of the acquired mesenchymal cell phenotype. Recent data have shown that sustained activation of an epithelial-mesenchymal transition results in progressive epigenetic changes in cells. These alterations induce heritable effects that maintain the mesenchymal state even after epithelial-mesenchymal transition-initiating signals are no longer present. ${ }^{8,11}$

\section{Primary breast cancer subtypes}

There are several subtypes of breast cancer, two of which are triple-negative and basal-like breast cancers. Triple-negative breast cancers are defined as tumors that lack expression of three receptors, ie, the estrogen receptor, progesterone receptor, and human epidermal growth factor receptor 2 (Her2). Basal-like breast cancers are characterized by the absence or low levels of estrogen receptor expression and the absence of Her2 overexpression, and also express many genes usually found in the basal or myoepithelial cells of the normal breast. Even though a majority of basal-like cancers are also triple-negative breast cancers and about $80 \%$ of triple-negative breast cancers are also basal-like, they are not synonymous. ${ }^{12-15} \mathrm{Up}$ to $20 \%$ of basal-like cancers express estrogen receptor or overexpress Her2. ${ }^{12}$ Triple-negative breast cancers encompass other molecular subtypes of breast cancer, including claudin-low tumors enriched with cells that have properties similar to stem cells and features of epithelial-mesenchymal transition, as well as interferon-rich tumors associated with considerably better prognosis than other triple-negative breast cancers. ${ }^{12-14}$ The triple-negative subtype accounts for $12 \%-17 \%$ of women with breast cancer and, as a group, these patients have a relatively poor outcome. ${ }^{12}$ Given the nature of these tumors, affected patients cannot be treated with endocrine therapy or therapies targeted against Her2. When Her2 is overexpressed, it is a target for trastuzumab (Herceptin ${ }^{\circledR}$ ), a humanized monoclonal antibody. Both triple-negative and basal-like breast cancers are more likely to metastasize to visceral organs, such as the lungs and brain, than they are to metastasize to bone.

At the genetic level, triple-negative and basal-like cancers are very heterogeneous. They account for almost $15 \%$ of all invasive breast cancers and usually have a high histological grade. ${ }^{12,16,17}$ Both types of breast cancer occur more frequently in young African-American and Hispanic women than in young women of other racial or ethnic groups. Women who develop a basal-like breast cancer often have certain features not shared by women without cancer; they reached menarche at an earlier age, they had a higher body mass index during the premenopausal years, had higher parity, and lower lifetime duration of breast-feeding. ${ }^{18,19}$ The risk of basal-like breast cancer rises with increasing parity and an increasing ratio of waist-to-hip circumference, indicating a complex relationship between genetic and societal factors. ${ }^{12,18}$ Basal-like breast cancer cells possess some phenotypic characteristics similar to those of breast stem cells and display gene expression patterns consistent with cells undergoing an epithelial-mesenchymal transition. Cancer stem cells are responsible for a distinct population of malignant cells with metastatic potential. They do not necessarily have to arise from the tissue stem cells, but can also come from other differentiated cancer cells that have acquired the property of self-renewal. Cancer cells from triple-negative and basallike breast cancers display a profile of cell-surface markers that is similar to that of breast cancer stem cells, such as the $\mathrm{CD} 44^{+} \mathrm{CD} 24^{-}$phenotype. ${ }^{12,20}$ It is currently unclear if all basal-like cancers are enriched with cancer stem cells or if they have a disproportionately high number of cells undergoing epithelial-mesenchymal transition, the process that enables cells to initiate invasion of distant organs.

Recent research has shown a link between the breast cancer 1 (BRCA1) pathway, basal-like breast cancers, and triple-negative breast cancers. More than $75 \%$ of tumors in 
women with a BRCA1 mutation also have a triple-negative phenotype, a basal-like phenotype, or both. ${ }^{12,16,17}$ Studies have found that basal-like breast cancers and tumors with a BRCA1 mutation have many characteristics in common compared with nonhereditary breast cancers or BRCA2related tumors. They both rarely have amplifications of the cyclic-D1 gene, they both express lower levels of p27, and express higher levels of S-phase kinase associated protein 2, cyclin E, fascin, caveolins 1 and 2, osteonectin, and caspase 3. ${ }^{12,21,22}$ One study found that both basal-like breast cancer and BRCA1-related breast cancer have a defect in maintaining normal chromosome $\mathrm{X}$ inactivation. This suggests that similarity between the two tumor types could lie in chromatin remodeling. ${ }^{12}$ Recent experiments have found that levels of BRCA1 protein may be lower in grade 3 tumors that do not express the estrogen receptor or progesterone receptor and possess a more basal-like phenotype than other types of breast cancer. This downregulation could be mediated by epigenetic changes. ${ }^{12}$ One study showed that mice deficient in both Brcal and p53, a tumor suppressor gene, in mammary epithelial cells developed tumors that were both triple-negative and basal-like in nature. The resulting tumors were very similar to those that occur in humans with a $B R C A 1$ mutation. This suggests that brcal plays a permissive role in the transition of undifferentiated breast cells to their more mature counterparts. ${ }^{12}$

Triple-negative and basal-like breast cancers show aggressive clinical behavior. The survival curve for patients with either type of breast cancer has a sharp decrease during the first 3-5 years after diagnosis. ${ }^{12}$ However, a distant relapse is much less common in later years. After 10 years, estrogen receptor-positive cancer patients are more likely to relapse than are those with estrogen receptor-negative tumors. ${ }^{12,23}$ There is some hope for a small subgroup of patients with either triple-negative or basal-like breast cancer. This population is markedly sensitive to chemotherapy and is associated with a good prognosis when treated with conventional chemotherapy agents. Some triple-negative and basallike breast cancers have a dysfunctional BRCA1 pathway, which may make them vulnerable to certain therapies.

Many studies have shown that angiogenesis is involved in breast cancer metastases to the brain. Brain metastatic tumors showed more angiogenesis but lower vascular permeability than did the primary breast cancer, suggesting that the cranial environment is leakage-resistant but proangiogenic. ${ }^{24,25}$ There are several essential factors for breast cancer metastasis, ie, vascular endothelial growth factor, matrix metalloproteinases (MMPs), and the chemokine receptor, CXCR4. One study confirmed that vascular endothelial growth factor is expressed nearly four times higher in primary breast cancer patients with brain metastases than in those without. ${ }^{26}$ Researchers speculated that vascular endothelial growth factor might enhance the transendothelial migration of tumor cells by downregulating the integrity of the endothelium. This was confirmed in several in vitro studies in which increased vascular endothelial growth factor expression was followed by an increase in the adhesion of tumor cells on the human brain microvascular endothelial cell monolayer. Brain metastatic lesions of several variants showed a higher vascular density and released significantly more vascular endothelial growth factor and interleukin- 8 when compared with the original cell line. ${ }^{24}$ One study used a vascular endothelial growth factor receptor-specific tyrosine kinase inhibitor to target endothelial cells and showed a reduction in angiogenesis and further restriction of brain metastatic growth. MMPs are zinc-dependent proteinases that play a key role in extracellular matrix degradation. In a breast cancer brain metastasis rat model, a micrometastasis showed a significantly higher expression of MMP2, MMP3, and MMP9, as well as an increase in MMP2 and MMP3 activities compared with the normal brain. The study showed that the development of a brain metastasis was significantly decreased with treatment using a selective synthetic MMP inhibitor. ${ }^{27}$ Another study confirmed that in human breast cancer cells overexpressing MMP2, there was a higher incidence of metastasis to the brain. ${ }^{28}$ An in vitro study showed that brain-seeking breast cancer cells had a higher total and active amount of MMP1 and MMP9, which provided the cells with a greater migration and invasion capacity. ${ }^{29}$ The addition of an MMP1 and/or MMP9 inhibitor decreased the amount of active proteinases as well as the migratory and invasive capabilities they provided to the cells.

It is known that malignant breast cancer cells express the chemokine receptor, CXCR4. Many studies have shown that the expression of CXCR4 is consistently higher in primary breast tumor cells than in normal breast epithelial cells. ${ }^{24}$ When these cancer cells invade the extracellular matrix and circulate in the blood and lymphatic vessels, they are attracted to their ligand, stromal cell-derived factor- $1 \alpha$. The malignant cells then leave the circulation and migrate into organs with large amounts of chemokines. Inside the parenchyma, the cancer cells proliferate, induce angiogenesis, and form metastatic tumors. An in vitro study showed that stromal cell-derived factor- $1 \alpha$ could induce blood vessel instability by increasing vascular permeability, resulting in the penetration of breast tumor cells through the human 
brain microvascular endothelial cell monolayers. In another study, anti-CXCR4 antibodies decreased transendothelial breast cancer migration as well as vascular permeability. ${ }^{30}$ Therefore, it is possible that Her2 could induce CXCR4 expression, and may interact with that chemokine in order to contribute to brain metastasis. ${ }^{24}$

Studies have shown that other chemokines and their receptors play an important role in the development and progression of brain metastases. The results from a recent study that analyzed a set of 142 axillary node-positive breast cancer patients suggested the presence of chemokine receptor, CX3CR1, and its ligand, fraktalkine, are significantly associated with brain metastases. ${ }^{31}$ Another chemokine and receptor pair that may be involved in breast cancer metastases is Slit and Robo. The Slit family of secreted proteins are Slit1, 2, and 3, and their corresponding Robo receptors are Robo1, 2, 3, and 4. They play an integral role in neuronal development because Slit proteins function in pioneer axon guidance for neurons in the brain and olfactory system; Slit1 is predominantly expressed in the nervous system. ${ }^{24}$ Studies have suggested that Slit/Robol signaling may be involved in the metastasis of breast cancer to the brain. ${ }^{24}$ An in vitro study showed that Slit2/Robol signaling was capable of inducing directed migration; Slit2 acted as a potent attractor for breast cancer cells expressing Robo1. Circulating Robo-expressing tumor cells, attracted to Slit, attached to the vascular endothelial cells in the brain. The increased activities of MMP9 and vascular endothelial growth factor then facilitated the penetration of malignant cells across the blood-brain barrier.

Another factor involved in cancer cell migration is store-operated calcium $\left(\mathrm{Ca}^{2+}\right)$ influx. A set of experiments by Yang et al determined that the function of two known regulators of $\mathrm{Ca}^{2+}$ influx was essential for breast cancer metastasis. The two regulators studied, ORAI1 and stromal interaction molecule 1 , have different roles in maintaining store-operated $\mathrm{Ca}^{2+}$ influx in nonexcitable cells; stromal interaction molecule 1 is a $\mathrm{Ca}^{2+}$ sensor and ORAI1 is part of a pore that enables store-operated $\mathrm{Ca}^{2+}$ entry. Previous studies have shown inhibiting store-operated channels suppressed serum-induced cell migration. ${ }^{32-36}$ Using small interfering RNAs against stromal interaction molecule 1 or ORAI1, Yang et al showed that these proteins are required for the migration of MDA-MB 231 human breast tumor cells in vitro. Furthermore, the cells showed significantly lower levels of metastatic growth. A pharmacological inhibitor of store-operated $\mathrm{Ca}^{2+}$ channels, SKF96365, reduced growth of 4T1 mouse breast cancer cells after orthotopic injections. ${ }^{37,38}$
Thus, stromal interaction molecule 1 and ORAI1 are potential new targets for the inhibition of breast cancer cell migration and metastasis.

There are several conventional treatments, such as corticosteroids, whole brain radiation therapy, surgical resection, stereotactic radiosurgery, and chemotherapy, as well as new techniques available for the treatment of brain metastases. Corticosteroids relieve symptoms by decreasing cerebral edema surrounding brain metastases, but have not shown large improvements in overall patient survival. Whole brain radiation therapy is the most common choice for patients with multiple brain metastases. ${ }^{24}$ It is also a popular option for patients with a solitary brain metastasis that does not qualify for either surgical resection or stereotactic radiosurgery. Whole brain radiation therapy is able to control neurological symptoms and has been shown to improve quality of life in about $75 \%-85 \%$ of patients. ${ }^{24}$ It can also prolong the mean survival of patients compared with corticosteroids alone. An advantage of surgical resection over both corticosteroids and whole brain radiation therapy is that it allows for pathological diagnosis. By decompressing the effect of the tumor mass, surgical resection may improve neurological symptoms as well and improved quality of life. Compared with supportive care alone, it has been shown to improve overall median survival. However, unless there is an obvious symptomatic lesion, surgical resection in patients with multiple brain metastases has limited use..$^{24,39-41}$ Stereotactic radiosurgery delivers focal radiation to areas smaller than $3.5 \mathrm{~cm}$, minimizing radiation exposure. It is less invasive than surgical resection and is given to patients who cannot tolerate surgery or have surgically inaccessible lesions. A study showed that the combination of stereotactic radiosurgery and whole brain radiation therapy significantly improved the overall survival of patients with a single brain metastasis, but provided no survival advantage for patients with multiple brain metastases. ${ }^{42}$ Chemotherapy is not a very useful treatment for single or multiple brain metastases because the tight junctions of the blood-brain barrier prevent the entry of most chemotherapeutic agents into the central nervous system. However, some drugs have shown promise when used in combination with radiation therapy. For example, efaproxiral can increase tumor oxidation and, therefore, increase radiation sensitivity. ${ }^{24,42,43}$ Studies have also shown an improvement in median survival when chemotherapy was used in combination with whole brain radiation therapy compared with whole brain radiation therapy alone. Several strategies for new delivery techniques are currently being tested in animal models. The placement of carmustine, an 
impregnated polymer wafer that allows for the slow release of chemotherapeutic agents in the resection cavity, has shown success in treating primary brain tumors..$^{24,42,44} \mathrm{It}$ is now being tested in metastasis cancers. Intracerebral microinfusion has shown effectiveness in animal studies but has not yet been tested in humans. ${ }^{24}$

However, therapies can indirectly and negatively influence the course and pattern of metastasis. Treatments targeted against systemic disease may ultimately favor the recurrence of cancer in specific organs. Several studies observed a rising incidence of brain metastasis in Her2positive breast cancer patients when they were treated with Herceptin. The US Food and Drug Administration approved Herceptin as an anticancer treatment in 1998. It has shown an improvement in disease-free and overall survival when delivered with cytotoxic chemotherapy to patients with Her2overexpressing metastatic breast cancer cells. However, some patients developed brain metastases, with an incidence of $25 \%-50 \%$, while responding to Herceptin over a period of 4-24 months. ${ }^{24}$ In certain breast cancer patients treated with Herceptin, Her2 overexpression is now known to be a predictive factor for central nervous system relapse. In an attempt to understand this paradoxical reaction, Musolino et al evaluated 54 patients with Her2-positive metastatic breast cancer. The patients studied had several different polymorphisms for the immunoglobulin $\mathrm{G}(\mathrm{IgG})$ fragment $\mathrm{C}$ receptor (Fc $\gamma \mathrm{R})$, an important protein involved in antibody-dependent cell-mediated cytotoxicity. The authors found a significant correlation between two specific Fc $\gamma \mathrm{R}$ polymorphisms and patients' response to Herceptin as well as progression-free survival. ${ }^{45}$ The results suggest that sequence variants in the genes encoding the Fcy receptors can be used to determine which patients are most likely to respond positively to treatment with Herceptin.

Chemotherapy is the current treatment available for women with triple-negative breast cancer; as a group, they have a worse outcome after chemotherapy than patients with other subtypes of breast cancer. As previously mentioned, there is a minority of women with triple-negative breast cancer whose tumors are extremely sensitive to chemotherapy. Neoadjuvant studies have shown treatment to be very effective in these patients. They show a complete pathological response and thus have an excellent outcome. Clinical trials are currently assessing the use of cisplatin and carboplatin to treat these sensitive triple-negative breast cancers. Initial findings suggest that neoadjuvant use of cisplatin results in high rates of complete pathological response in patients with breast cancer who have BRCA1 mutations and perhaps in patients with triple-negative cancer. ${ }^{12,46,47}$ Overexpression of epidermal growth factor receptor is more common in triple-negative breast cancer than in other subtypes. The use of cetuximab, a monoclonal antibody, targeted against the epidermal growth factor receptor, is currently being studied in combination with carboplatin. However, triple-negative and basal-like breast cancers often display abnormalities in phosphatase and tensin homolog, which is frequently associated with resistance to antiepidermal growth factor receptor therapies. A recent clinical target in triple-negative breast cancer is the enzyme PARP (poly[adenosine diphosphate-ribose] polymerase), which is involved in base-excision repair after DNA damage. ${ }^{12}$ Inhibitors of PARP have shown very encouraging clinical activity in early trials with BRCA mutation tumors and sporadic triple-negative cancers. ${ }^{48,49}$ In a recent randomized Phase II trial, a PARP inhibitor used in combination with chemotherapy resulted in statistically significant improvements in the rate of tumor regression, median progressionfree survival, and median overall survival.

\section{Breast to brain metastases}

Breast to brain metastases appear in either the parenchyma or along the leptomeninges. The majority of tumors occur in the parenchyma and follow a vascular distribution mainly through hematogenous spread..$^{50}$ In over $50 \%$ of patients with melanoma or lung cancer, parenchymal metastases occur simultaneously with leptomeningeal metastases. ${ }^{51}$ Clinical symptoms of parenchymal brain metastases include headache, mental status change, cognitive disturbances, and other manifestations that depend on the location of the lesion. Leptomeningeal tumors are less common but can arise through multiple pathways including hematogenous spread, infiltration from vertebral metastases via the venous plexus, and extension along nerves or perineural lymphatics. ${ }^{52}$ They need more than three years to develop in patients with melanoma and breast cancer. ${ }^{53}$ Clinical symptoms of leptomeningeal metastases tend to be nonlocalizing, such as pain, headache, and cranial neuropathies. Two types of neuroimaging are popular for detecting brain metastases, ie, gadolinium-enhanced magnetic resonance imaging and contrast-enhanced computed tomography. Magnetic resonance imaging is preferred over computed tomography because it is more sensitive for identifying parenchymal and leptomeningeal metastases. ${ }^{54,55}$ Cerebrospinal fluid cytology has a higher specificity than that of magnetic resonance imaging and can be used to detect leptomeningeal metastases as well. ${ }^{24,52}$ 
Studies have revealed that directly or indirectly, an individual's genetic background can determine metastasis susceptibility in breast cancer. ${ }^{56}$ Population genetics studies have delineated inherited tendencies to develop cancer and suggested the existence of alleles that predispose individuals to breast cancer metastasis. ${ }^{56,57} \mathrm{~A}$ study combining gene expression analysis and population genetics revealed that tumors from premenopausal African-American patients expressed a gene signature that represents the basal subtype of breast cancer with a higher prevalence than did those from Caucasian patients. This polygenic signature correlates with aggressive metastatic relapse. Using a quantitative trait locus analysis and multiple cross-mapping study, researchers identified a single nucleotide polymorphism in SIPA1, a GTPase-activating protein that inhibits RAP1 and RAP2 activity, which determined metastatic susceptibility. This single nucleotide polymorphism resulted in an amino acid substitution that hinders GTPase-activating protein function, attenuating pulmonary metastasis. Polymorphisms of this kind have been reported in human breast cancer samples and correlate with poor prognosis. The pleiotropic effects of these genetic and allelic factors might contribute to multiple steps in malignant progression, from the aggressiveness of local tumor cells to their dissemination and the onset of distant organ metastasis.

A member of the epidermal growth factor receptor family, Her2/ErbB2/neu, plays an essential role in breast cancer to brain metastasis and overall patient survival. The ERBB2 gene encodes a transmembrane receptor tyrosine kinase that is elevated in $20 \%-30 \%$ of human breast cancers. ${ }^{24,58-60}$ The elevation results from genomic amplification of the ErbB2 proto-oncogene and transcriptional upregulation of the ErbB2 promoter. ${ }^{24}$ Patients with overexpression of Her 2 had a worse overall survival because this predicts tamoxifen resistance in the primary tumor. Furthermore, Her2 overexpression has been shown to be associated with the brain metastatic phenotype. The correlation between Her2 overexpression in primary breast cancers and subsequent brain metastases is 97\%. ${ }^{61}$ Thus, Her2 amplification is an important risk factor for brain metastasis.

Upon activation, Her2 phosphorylates many downstream molecules that activate a variety of signaling cascades. By activating different pathways, Her2 causes changes in gene expression levels for proteins implicated in cell growth, survival, and metastases. Overexpression of Her2 has been found to increase membrane degradation by activating the transcription and enhancing the secretion of MMP9. Overexpression of Her2 also increases the invasiveness of breast cancer cells by upregulating the chemokine receptor, CXCR4 . Breast cancer cells that express high levels of CXCR4 increase the permeability of brain endothelial cells, facilitating their invasion into the parenchyma. If a therapeutic drug can penetrate the blood-brain barrier, Her2 directed therapy could be targeted against Her2-overexpressing breast cancer cells growing in the brain. ${ }^{24}$ Lapatinib is an irreversible inhibitor of the Her2 tyrosine kinase that can cross the blood-brain barrier. In a study to determine its effects, responses were observed in two of 39 patients. ${ }^{39}$

\section{Role of blood-brain barrier in metastasis}

Of all the central nervous system barriers, the blood-brain barrier exerts the greatest control over the immediate microenvironment of brain cells. Early histological studies have shown that brain capillaries are surrounded by or closely associated with several cell types, including the perivascular endfeet of astrocytic glia, pericytes, microglia, and neuronal processes. Recently, pericytes, perivascular macrophages, and neurons have been shown to contribute to induction of the blood-brain barrier. Astrocytes can regulate many features of the blood-brain barrier, such as expression and polarized location of transporters and specialized enzyme systems, resulting in tighter tight junctions. ${ }^{62}$ They tightly ensheath the vessel wall and seem to be critical for the induction and maintenance of the endothelial barrier. ${ }^{2}$ Astrocytes can secrete a range of chemical agents, such as transforming growth factor- $\beta$, glial-derived neurotrophic factor, basic fibroblast growth factor, and angiopoietin 1, which induce aspects of the blood-brain barrier phenotype in endothelial cells in vivo. ${ }^{62-64}$

Many features of the blood-brain barrier phenotype are subject to change or modulation. Astroglia can release chemical factors and signals that modify the permeability of the brain endothelium. ${ }^{2}$ The transcription factor, nuclear factor-kappa B, can alter tight junction protein expression and thus regulates blood-brain barrier permeability. ${ }^{62}$ Traditionally, resistance of brain metastasis to chemotherapy has been attributed to the blood-brain barrier. However, recent studies have revealed that the blood-brain barrier is not intact in larger metastases because the metastatic tumor cells release vascular endothelial growth factor, ie, the vascular permeability factor. The blood-brain barrier remains intact in and around experimental brain metastases smaller than $0.25 \mathrm{~mm}$ in diameter. ${ }^{65}$ More than $70 \%$ of brain metastasis cases exhibit enhanced lesions on magnetic resonance imaging due to leakage of contrast agents from blood vessels, 
indicating blood-brain barrier permeability. ${ }^{66,67}$ With larger tumors, the tight junctions between the endothelial cells become stretched out. Brain metastasis might involve an active crosstalk between the cancer and stromal cells, as suggested by the range of unique cell types that constitute the brain parenchyma and their anatomical organization. ${ }^{68,69}$

Astrocytes are the predominant cells in the brain and maintain homeostasis of the brain microenvironment. Under certain pathological conditions, such as trauma, ischemia, and neurodegenerative disease, astrocytes can become activated, as indicated by the upregulation of glial fibrillary acidic protein. These reactive astrocytes have been shown to protect neurons from injury-induced apoptosis..$^{51,66,70}$ Reactive astrocytes can also protect melanoma cells in brain metastases from cytotoxicity due to chemotherapeutic drugs. ${ }^{66}$ An experiment by Lin et al studied the sensitivity of melanoma cells to chemotherapeutic agents when cocultured with mouse astrocytes or fibroblasts. Their results revealed that astrocytes, not fibroblasts, reduced apoptosis in human melanoma cells treated with various chemotherapeutic drugs. The chemotherapeutic effect was dependent on physical contact and gap junctional communication between astrocytes and tumor cells. The results of these recent experiments showed that the astrocytes sequestered calcium from the cytoplasm of tumor cells. Calcium is one of the most important second messengers transmitted through gap junctional communication channels. It has been shown to play a causal role in cell death. Melanoma cells incubated with chemotherapeutic agents showed an increase in cytoplasmic calcium followed by the presence of fragmented DNA, a characteristic of apoptosis. A flow cytometry analysis of cytoplasmic $\mathrm{Ca}^{2+}$ in paclitaxel-treated tumor cells revealed that coculture with astrocytes significantly reduced cytoplasmic calcium levels when compared with tumor cells incubated with paclitaxel alone. These data implicate calcium sequestration through gap junctional communication channels as a key mechanism by which astrocytes protect tumor cells from chemotherapy. Thus, brain tumors can harness the neuroprotective effects of reactive astrocytes for their own survival. Successful treatment of brain metastasis will require chemotherapy in combination with agents that selectively interfere with gap junctional communication channels. Targeted therapies could include glial fibrillary acidic protein antibodies to tumor-associated astrocytes or short nucleotide sequences complementary to glial fibrillary acidic protein mRNA. ${ }^{66}$ The results from Lin et al present a previously unrecognized mechanism for drug resistance in brain metastases that will have important clinical implications.
The blood-brain barrier is composed of xenobiotic transporters that expel substrates from the brain into the cerebrospinal fluid and systemic circulation. These transporters also extrude drugs and toxins if they gain entry into the cytoplasm of brain endothelial cells. It is expected that lipid-soluble drugs would readily cross the blood-brain barrier, but studies have found that many of them have a lower permeability than predicted by their lipid solubility. These drugs happen to be substrates for the drug efflux transporters, which are also present in the blood-cerebrospinal fluid barrier, the activity of which very efficiently removes drugs from the central nervous system and so limits brain uptake. Studies have identified numerous efflux transporters that comprise the blood-brain barrier, such as P-glycoprotein, members of the multidrug resistance protein (MDR) family, and members of the organic cation and anion transporter families.

The MDR gene, $m d r 1$, encodes P-glycoprotein (or ABCB1). It was initially discovered as a highly expressed protein in multidrug resistant tumor cell lines. Its expression in the brain has been found in many species, including humans, rats, and primates. ${ }^{2,71}$ In the brain capillary, $\mathrm{P}$-glycoprotein is mainly expressed at the luminal membrane where it serves as an efflux pump, restricting or preventing entry of substrates into the brain parenchyma. Interestingly, many of the chemotherapeutic agents used in clinical practice were discovered to be substrates for P-glycoprotein. In Mdrl knockout mice, researchers observed an increase in the brain of P-glycoprotein substrates from the circulation compared with wild-type mice. Similarly, animals treated with inhibitors of P-glycoprotein showed increased amounts of P-glycoprotein substrates in the central nervous system. Mice with a xenograft glioblastoma tumor were given paclitaxel and a P-glycoprotein inhibitor and showed an increased brain concentration of paclitaxel and improved tumor response compared with animals given paclitaxel alone. This increased penetration of drugs after P-glycoprotein inhibition has been confirmed in a human study.

There are other important transporters involved in forming the blood-brain barrier, including MDR1 through MDR9, all members of the ABCC family of transporters. The transporter ABCG2 (or BCRP) was initially named the breast cancer-resistant protein because it was first discovered in a chemotherapy-resistant breast cancer cell line. It has been detected in the capillary endothelial cells of the brain in humans as well as in other species, and is mainly expressed on the luminal surface. Based on mRNA expression levels, ABCG2 may be even more strongly expressed than either P-glycoprotein or MDR1.,72 In one study, Bcrp1 
knockout mice had a 2.5-fold increase in brain concentration of a substrate for ABCG2 compared with wild-type mice. ${ }^{73}$ Similarly, another study found that coadministration of an ABCG2 substrate and inhibitor resulted in a 4.2-fold increase in brain penetration. ${ }^{74}$ Organic anion and cation transporter families have also been found in the brain endothelium. They typically exchange anions and cations, respectively, across concentration gradients from the blood to the brain, or vice versa. The exact location of transporters from the organic anion transporter and organic cation transporter families as well as those from the organic anion-transporting polypeptide family is unclear. ${ }^{2}$ Studies determining the role of organic anion transporters and organic anion-transporting polypeptides in forming the blood-brain barrier are difficult to perform, given the overlapping substrate specificity of organic anion transporters and organic anion-transporting polypeptides with $\mathrm{ABC}$ transporters. ${ }^{2}$

Primary and metastatic tumors produce changes in the expression of xenobiotic transporters. The expression level of P-glycoprotein in blood vessels supplying melanoma central nervous system metastases was only $5 \%$ of that seen in normal brain tissue., ${ }^{2,75}$ Similarly, the vasculature around central nervous system lung metastases had just $40 \%$ of the P-glycoprotein expression found in normal brain vasculature. ${ }^{2,75}$ Two different studies found conflicting levels of P-glycoprotein expression in the vasculature of malignant gliomas. Becker et al found diminished expression compared with normal brain vasculature, ${ }^{76}$ while Toth et al found no difference between tumor vasculature and normal tissue. ${ }^{77}$ Haga et al also found no difference in the expression level of P-glycoprotein and MDR2 between normal brain and malignant glioma cells. ${ }^{78}$ However, they did find increased expression of MDR1 and MDR3 in the endothelial cells forming the vasculature around tumor sites.

\section{Mesenchymal-epithelial transition}

Distant metastases derived from primary cancers are largely composed of cancer cells with an epithelial phenotype closely resembling that of cancer cells in the primary tumor. In order to disseminate, cancer cells must pass through an epithelial-mesenchymal transition and acquire mesenchymal features, but the resulting metastases look like the primary cancers at the histopathological level. Following metastatic spread and colonization, a mesenchymal-epithelial transition usually converts disseminated mesenchymal cancer cells back to a more differentiated epithelial cell state. In the absence of signals that actively promote the induction and continued expression of an epithelial-mesenchymal transition, many normal and neoplastic cells will revert back to the epithelial state through a transcriptional default mechanism. ${ }^{8}$ For example, several studies have shown that the DNA methylation status of the CDH1 promote, ie, the gene for E-cadherin, an essential cell adhesion molecule, varies at different stages of the metastatic process. In primary breast cancers, the tumor cells that undergo transient hypermethylation and silencing of $\mathrm{CDH} 1$ expression are more invasive and metastatic. ${ }^{8}$ However, E-cadherin expression is reinduced in metastases and is accompanied by demethylation of the CDH1 promoter. ${ }^{8,79,80}$ Epithelial-mesenchymal transitions and their converses, ie, mesenchymal-epithelial transitions, are central regulators of cellular plasticity in cancers. They have important roles in therapeutic resistance, tumor recurrence, and metastatic progression.

\section{Difficulty with treatment}

Primary brain malignancies are intrinsically resistant to most chemotherapies, and metastases are often associated with resistance to treatment. This might be due to cell-intrinsic mechanisms, such as genetic alterations, that confer drug resistance after a period of therapeutic response. ${ }^{68}$ Cancer cells in the central nervous system could be shielded by the blood-brain barrier from drug delivery or protected by survival signals from the host microenvironment. The unique structure of the blood-brain barrier limits the effectiveness of current treatments. However, there is a breakdown of the blood-brain barrier in brain tumors. During inflammation, certain released agents have the ability to increase the permeability of the brain endothelium, as shown in Figure $1 .{ }^{62}$ Bradykinin acts on endothelial bradykinin $\mathrm{B}_{2}$ receptors to raise intracellular $\mathrm{Ca}^{2+}$ concentrations and open tight junctions. Tumor necrosis factor- $\alpha$ can further increase blood-brain barrier permeability by directly acting on the endothelium and by initiating interleukin-1 $\beta$ release from astrocytes via an immunoregulatory loop. ${ }^{62,81,82}$ The bloodbrain barrier is greatly impaired only in larger tumors in terms of transporter expression and function, as well as in terms of endothelium permeability. This allows a sufficient amount of chemotherapy to reach the tumor and effect a response. In smaller aggregates of metastatic tumor cells, the disruption of the blood-brain barrier is less significant. Because a smaller amount of drug reaches these micrometastases, they can grow, develop neovascular structures, and ultimately reach a clinically significant size.

There are three general methods in use to increase drug delivery to the brain. The first is the administration of chemotherapy agents directly into the central nervous system 


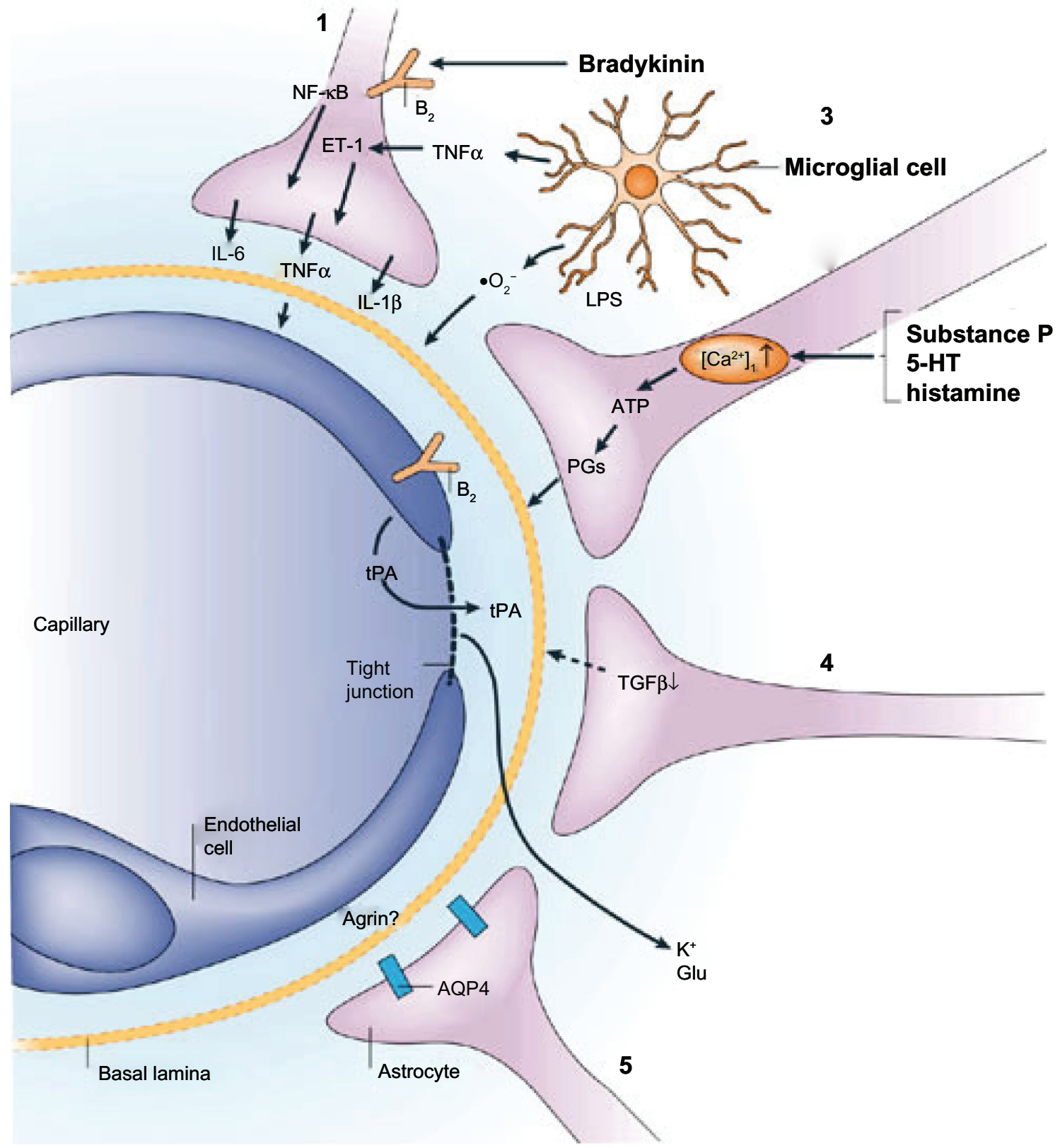

Figure I Examples of astroglial-endothelial signaling in infection or inflammation, stroke or trauma, leading to opening of the blood-brain barrier and disturbance of brain function. Bradykinin, produced during inflammation in stroke or brain trauma, acts on endothelial and astroglial bradykinin $\mathrm{B}_{2}$ receptors, leading to an increase in the concentration of intracellular $\mathrm{Ca}^{2+}$. In astrocytes, this can trigger the production of IL-6 through activation of nuclear factor- $\mathrm{KB}$.' (I). Bradykinin, substance $\mathrm{P}$, 5 - $\mathrm{HT}$ (serotonin) and histamine acting on astrocytes can lead to the formation of ATP and PGs, with effects on vascular tone and endothelial permeability (2) by mechanisms that are known to involve endothelium. Lipopolysaccharide (LPS), formed in infections, leads to the release from microglia of TNF $\alpha$, IL-I $\beta$, and reactive oxygen species (including $\mathrm{O}_{2}{ }^{-}$), all of which have the ability to open the blood-brain barrier (3). Astrocytes downregulate tPA production via TGF $\beta$, but there is still sufficient tPA to open the blood-brain barrier, leading to an influx of tPA from the blood (4). Following disruption of the blood-brain barrier involving a decrease in agrin expression, $\mathrm{K}^{+}$and $\mathrm{Glu}$ from the blood can reach the brain extracellular space. AQP4 is upregulated on the astroglial endfeet, leading to astroglial swelling (5). Reprinted by permission from Macmillan Publishers Ltd: Nature Publishing Group ${ }^{62}$, copyright 2006.

Abbreviations: IL, interleukin; NF- $\mathrm{BB}$, nuclear factor- $\mathrm{KB}$; 5-HT, 5-hydroxytryptamine; PGs, prostaglandins; TNF $\alpha$, tumor necrosis factor- $\alpha$; tPA, tissue plasminogen activator; TGF $\beta$, transforming growth factor- $\beta$; Glu, glutamate; AQP4, aquaporin 4; ETI, endothelin I.

using Ommaya reservoirs, intrathecal injections, intra-arterial injections, or high-dose systemic therapy. Although this approach has found success, toxicities can be significant. ${ }^{2}$ The second method is to disrupt the blood-brain barrier and then administer chemotherapy. In order to increase the central nervous system penetration of chemotherapy agents, the blood-brain barrier is often disrupted with hypertonic solutions, such as mannitol. Another agent that disrupts the blood-brain barrier is RMP-7, a synthetic analog of the peptide, bradykinin. It is specific for the bradykinin-P2 receptor, which participates in the modulation of tight junctions in brain endothelial cells. In preclinical models, 
RMP-7 has been found to increase the brain concentration of a chemotherapy agent using intracarotid or intravenous administration. ${ }^{2,83}$ Unfortunately, this activity was not observed in several Phase II trials. The third approach is to deliver chemotherapy drugs with an agent that inhibits the drug transporters of the blood-brain barrier. In preclinical trials, researchers found a higher central nervous system penetrance of chemotherapy agents after inhibition of ABCG2. Over the past two decades, researchers have been looking to develop agents that inhibit P-glycoprotein at the cellular level and, thus, increase intracellular concentrations of toxic chemotherapy agents in resistant tumors. However, clinical trials have been disappointing in that promising Phase II trials were followed by negative Phase III trials. However, all hope is not lost. In animal models, the administration of P-glycoprotein inhibitors has been found to increase intracranial concentrations of chemotherapy agents.

There are several creative strategies to deliver drugs across the blood-brain barrier currently being tested in both in vitro and in vivo models. The human brain microvascular endothelial cell is a widely used in vitro model system that mimics the in vivo human blood-brain barrier. With this model, researchers have used nanoparticles to facilitate the entry of drugs into the brain parenchyma. They encapsulated chemotherapy agents and other drugs in $250 \mathrm{~nm}$ diameter nanoparticles using poly(butyl)cyanoacrylate. ${ }^{2}$ The nanoparticles were then endocytosed by the blood-brain barrier endothelium and the drug was free to diffuse into the brain parenchyma. Using animal models, researchers tested the delivery of drugs across the blood-brain barrier by tagging or attaching liposome-containing drugs to an antibody that recognizes receptors along the endothelium. Endogenous large-molecule peptides, such as transferrin, insulin, and leptin, cross the blood-brain barrier via receptor-mediated transport by attaching to such receptors. The monoclonal antibody, OX26, which recognizes the transferrin receptor, was attached to a liposome containing digoxin, allowing the drug to cross the blood-brain barrier. ${ }^{2}$ Huwyler et al used the OX26 immunoliposome to transport daunorubicin in an in vivo animal model and found increased brain delivery and higher concentrations of the drug by a factor of four log values when compared with delivery without this vehicle. ${ }^{84}$ Another approach is to deliver chemotherapy drugs with an agent that selectively utilizes the influx transporters in the blood-brain barrier that move substances from the circulation into the brain parenchyma. This is an important strategy, not only to circumvent the efflux capacity of the blood-brain barrier, but also to increase drug delivery to brain tumors. ${ }^{2}$

\section{Potential therapeutic interventions}

Micrometastases often remain after conventional surgery, radiotherapy, and/or chemotherapy. By targeting the many diverse signal transduction pathways that contribute to the invasive and metastatic properties of cancer cells, therapeutic interventions have the potential to block tumor progression and prevent metastasis. ${ }^{85}$ Given that epithelial-mesenchymal transitions are associated with a poor clinical outcome in multiple tumor types, it is essential to understand the processes that trigger these transitions in order to prevent them. In several studies, an epithelial-mesenchymal transition has been shown to result in cancer cells with stem cell-like characteristics, such as invasion of the parenchyma and resistance to certain therapeutic interventions. Cancer cells with stem cell characteristics are enriched in tumors remaining after standard chemotherapeutic treatments. ${ }^{8,86}$ Thus, inhibition of epithelial-mesenchymal transition is an attractive therapeutic approach. However, the complexity of the signaling networks involved that regulate the induction of epithelial-mesenchymal transitions and the reversibility of the acquired mesenchymal phenotype pose significant challenges to researchers. ${ }^{8}$ It is not known when therapies that could inhibit this transition should be initiated. ${ }^{8,87,88}$ It is also unknown which pathways should be inhibited to be most effective while causing minimal toxicity in normal tissues. The process is further complicated by the similarities between epithelial-mesenchymal transition and normal stem cell programs. ${ }^{8}$

Previous studies have shown that, to produce brain metastasis, tumor cells must reach the vasculature of the brain, attach to the endothelial cells of the microvasculature, extravasate into the parenchyma, proliferate in response to growth factors, and induce the formation of new blood vessels. Vascular endothelial growth factor stimulates the proliferation and migration of endothelial cells and induces expression of MMPs and plasminogen activity. Overexpression of vascular endothelial growth factor in tumor cells enhances tumor growth and metastasis by stimulating vascularization and increasing microvessel density. Studies outlining the important role of vascular endothelial growth factor in the progressive growth of brain metastases have shown that vascular endothelial growth factor expression is necessary, but not sufficient, for the production of brain metastases.

Given the important role of vascular endothelial growth factor in cancer progression and neoangiogenesis, several vascular endothelial growth factor antagonists have been approved by the Food and Drug Administration. They 
increase survival in patients with metastatic breast and colorectal cancers when combined with chemotherapy. ${ }^{89,90}$ Despite their positive effects on established localized tumors, inhibition of angiogenesis can result in increased tumor invasion and metastasis. ${ }^{91,92}$ In a glioblastoma multiforme mouse model, treatment with sunitinib, a vascular endothelial growth factor receptor and platelet-derived growth factor receptor kinase inhibitor, or SU10944, a vascular endothelial growth factor receptor-selective kinase inhibitor, increased invasion. The data suggest that vascular endothelial growth factor inhibitors might precondition the host microenvironment, making it favorable for metastasis. ${ }^{91,92}$ Therefore, the proper timing of antiangiogenic therapy needs to be determined. It is also unclear whether combining these agents with chemotherapy or other treatments can counteract their unfavorable effects.

\section{Conclusion}

The need to understand the biology behind each step in the metastatic process becomes increasingly important as clinical oncology moves toward personalized medicine. Given that there are many different dimensions involved in the formation of a tumor and its metastasis, treatment will need to be specific to the individual and will have to include a combination of therapies. Treatments should be targeted to different stages of the metastatic cascade as well as the many signal transduction pathways and receptors involved. An analysis of certain transcription factors or the status of specific gene promoters can help researchers determine the time at which different therapies should be implemented.

Using a fusion of experimental techniques, several researchers have exposed populations that are susceptible to certain types of cancers. These groups should be encouraged to participate in early screening and take preventive measures to protect themselves against disease. Researchers have also identified several populations of breast cancer patients that are more sensitive to treatment than others. Therapies should be aggressively targeted against the dysfunctional pathways inherent in those tumors. Future studies may also need to follow a genetics approach to delineate other vulnerable groups and actively target them.

The success of future studies will rest on the researchers' understanding of the dynamic relationships involved in breast to brain metastases. With so many questions about metastases left unanswered, any treatment that immobilizes a specific pathway, receptor, or signaling molecule in the metastatic cascade will be critical in the fight to find a cure for metastatic brain cancer. However, in order to realize the ultimate goal of conquering cancer, all therapies must target and exploit the weaknesses within each individual tumor.

\section{Disclosure}

The authors report no conflicts of interest in this work.

\section{References}

1. Jemal A, Bray F, Center MM, Ferlay J, Ward E, Forman D. Global cancer statistics. CA Cancer J Clin. 2011;61(2):69-90.

2. Deeken JF, Loscher W. The blood-brain barrier and cancer: transporters, treatment, and Trojan horses. Clin Cancer Res. 2007;13(6): 1663-1674.

3. Schouten LJ, Rutten J, Huveneers HA, Twijnstra A. Incidence of brain metastases in a cohort of patients with carcinoma of the breast, colon, kidney, and lung and melanoma. Cancer. 2002;94(10):2698-2705.

4. Patel JK, Didolkar MS, Pickren JW, Moore RH. Metastatic pattern of malignant melanoma. A study of 216 autopsy cases. Am J Surg. 1978; 135(6):807-810.

5. Neman J, Somlo G, Jandial R. Classification of genomic changes in breast cancer brain metastasis. Neurosurgery. 2010;67(2):N18-N19.

6. Gril B, Evans L, Palmieri D, Steeg PS. Translational research in brain metastasis is identifying molecular pathways that may lead to the development of new therapeutic strategies. Eur J Cancer. 2010;46(7): 1204-1210.

7. Lin NU, Winer EP. Brain metastases: the HER2 paradigm. Clin Cancer Res. 2007;13(6):1648-1655.

8. Polyak K, Weinberg RA. Transitions between epithelial and mesenchymal states: acquisition of malignant and stem cell traits. Nat Rev Cancer. 2009;9(4):265-273.

9. Thiery JP, Sleeman JP. Complex networks orchestrate epithelialmesenchymal transitions. Nat Rev Mol Cell Biol. 2006;7(2):131-142.

10. Peinado H, Olmeda D, Cano A. Snail, Zeb and bHLH factors in tumour progression: an alliance against the epithelial phenotype? Nat Rev Cancer. 2007;7(6):415-428.

11. Dumont N, Wilson MB, Crawford YG, Reynolds PA, Sigaroudinia M, Tlsty TD. Sustained induction of epithelial to mesenchymal transition activates DNA methylation of genes silenced in basal-like breast cancers. Proc Natl Acad Sci U S A. 2008;105(39):14867-14872.

12. Foulkes WD, Smith IE, Reis-Filho JS. Triple-negative breast cancer. N Engl J Med. 2010;363(20):1938-1948.

13. Weigelt B, Baehner FL, Reis-Filho JS. The contribution of gene expression profiling to breast cancer classification, prognostication and prediction: a retrospective of the last decade. J Pathol. 2010;220(2): 263-280.

14. Sotiriou C, Pusztai L. Gene-expression signatures in breast cancer. N Engl J Med. 2009;360(8):790-800.

15. Kreike B, van Kouwenhove M, Horlings H, et al. Gene expression profiling and histopathological characterization of triple-negative/ basal-like breast carcinomas. Breast Cancer Res. 2007;9(5):R65.

16. Rakha EA, Reis-Filho JS, Ellis IO. Basal-like breast cancer: a critical review. J Clin Oncol. 2008;26(15):2568-2581.

17. Reis-Filho JS, Tutt AN. Triple negative tumours: a critical review. Histopathology. 2008;52(1):108-118.

18. Millikan RC, Newman B, Tse CK, et al. Epidemiology of basal-like breast cancer. Breast Cancer Res Treat. 2008;109(1):123-139.

19. Yang XR, Sherman ME, Rimm DL, et al. Differences in risk factors for breast cancer molecular subtypes in a population-based study. Cancer Epidemiol Biomarkers Prev. 2007;16(3):439-443.

20. Morrison BJ, Schmidt CW, Lakhani SR, Reynolds BA, Lopez JA. Breast cancer stem cells: implications for therapy of breast cancer. Breast Cancer Res. 2008;10(4):210.

21. Turner N, Tutt A, Ashworth A. Hallmarks of 'BRCAness' in sporadic cancers. Nat Rev Cancer. 2004;4(10):814-819.

22. Turner NC, Reis-Filho JS, Russell AM, et al. BRCA1 dysfunction in sporadic basal-like breast cancer. Oncogene. 2007;26(14):2126-2132. 
23. Cheang MC, Voduc D, Bajdik C, et al. Basal-like breast cancer defined by five biomarkers has superior prognostic value than triple-negative phenotype. Clin Cancer Res. 2008;14(5):1368-1376.

24. Cheng X, Hung MC. Breast cancer brain metastases. Cancer Metastasis Rev. 2007;26(3-4):635-643.

25. Monsky WL, Mouta Carreira C, Tsuzuki Y, Gohongi T, Fukumura D, Jain RK. Role of host microenvironment in angiogenesis and microvascular functions in human breast cancer xenografts: mammary fat pad versus cranial tumors. Clin Cancer Res. 2002;8(4):1008-1013.

26. Linderholm B, Grankvist K, Wilking N, Johansson M, Tavelin B, Henriksson R. Correlation of vascular endothelial growth factor content with recurrences, survival, and first relapse site in primary node-positive breast carcinoma after adjuvant treatment. J Clin Oncol. 2000;18(7):1423-1431.

27. Mendes O, Kim HT, Stoica G. Expression of MMP2, MMP9 and MMP3 in breast cancer brain metastasis in a rat model. Clin Exp Metastasis. 2005;22(3):237-246.

28. Tester AM, Waltham M, Oh SJ, et al. Pro-matrix metalloproteinase-2 transfection increases orthotopic primary growth and experimental metastasis of MDA-MB-231 human breast cancer cells in nude mice. Cancer Res. 2004;64(2):652-658.

29. Stark AM, Anuszkiewicz B, Mentlein R, Yoneda T, Mehdorn HM, Held-Feindt J. Differential expression of matrix metalloproteinases in brain- and bone-seeking clones of metastatic MDA-MB-231 breast cancer cells. J Neurooncol. 2007;81(1):39-48.

30. Lee BC, Lee TH, Avraham S, Avraham HK. Involvement of the chemokine receptor CXCR4 and its ligand stromal cell-derived factor 1 alpha in breast cancer cell migration through human brain microvascular endothelial cells. Mol Cancer Res. 2004;2(6):327-338.

31. Andre F, Cabioglu N, Assi H, et al. Expression of chemokine receptors predicts the site of metastatic relapse in patients with axillary node positive primary breast cancer. Ann Oncol. 2006;17(6):945-951.

32. Komuro H, Rakic P. Modulation of neuronal migration by NMDA receptors. Science. 1993;260(5104):95-97.

33. Lee J, Ishihara A, Oxford G, Johnson B, Jacobson K. Regulation of cell movement is mediated by stretch-activated calcium channels. Nature. 1999;400(6742):382-386.

34. Nishiyama M, Hoshino A, Tsai L, et al. Cyclic AMP/GMP-dependent modulation of $\mathrm{Ca}^{2+}$ channels sets the polarity of nerve growth-cone turning. Nature. 2003;423(6943):990-995.

35. Marks PW, Maxfield FR. Transient increases in cytosolic free calcium appear to be required for the migration of adherent human neutrophils. J Cell Biol. 1990;110(1):43-52.

36. Yang $\mathrm{S}$, Huang XY. $\mathrm{Ca}^{2+}$ influx through $\mathrm{L}$-type $\mathrm{Ca}^{2+}$ channels controls the trailing tail contraction in growth factor-induced fibroblast cell migration. J Biol Chem. 2005;280(29):27130-27137.

37. Yang S, Zhang JJ, Huang XY. Orai1 and STIM1 are critical for breast tumor cell migration and metastasis. Cancer Cell. 2009;15(2): 124-134.

38. McCarthy N. Calcium influx is moving. Nat Rev Cancer. 2009;9: 230-231.

39. Lin NU, Bellon JR, Winer EP. CNS metastases in breast cancer. J Clin Oncol. 2004;22(17):3608-3617.

40. Bindal RK, Sawaya R, Leavens ME, Lee JJ. Surgical treatment of multiple brain metastases. J Neurosurg. 1993;79(2):210-216.

41. Hazuka MB, Burleson WD, Stroud DN, Leonard CE, Lillehei KO, Kinzie JJ. Multiple brain metastases are associated with poor survival in patients treated with surgery and radiotherapy. J Clin Oncol. 1993; 11(2):369-373.

42. Peacock KH, Lesser GJ. Current therapeutic approaches in patients with brain metastases. Curr Treat Options Oncol. 2006;7(6):479-489.

43. Suh JH, Stea B, Nabid A, et al. Phase III study of efaproxiral as an adjunct to whole-brain radiation therapy for brain metastases. $J$ Clin Oncol. 2006;24(1):106-114.

44. Valtonen S, Timonen U, Toivanen P, et al. Interstitial chemotherapy with carmustine-loaded polymers for high-grade gliomas: a randomized double-blind study. Neurosurgery. 1997;41(1):44-48.
45. Musolino A, Naldi N, Bortesi B, et al. Immunoglobulin G fragment $\mathrm{C}$ receptor polymorphisms and clinical efficacy of trastuzumab-based therapy in patients with HER-2/neu-positive metastatic breast cancer. J Clin Oncol. 2008;26(11):1789-1796.

46. Byrski T, Huzarski T, Dent R, et al. Response to neoadjuvant therapy with cisplatin in BRCA1-positive breast cancer patients. Breast Cancer Res Treat. 2009;115(2):359-363.

47. Silver DP, Richardson AL, Eklund AC, et al. Efficacy of neoadjuvant cisplatin in triple-negative breast cancer. J Clin Oncol. 2010;28(7): 1145-1153.

48. Fong PC, Boss DS, Yap TA, et al. Inhibition of poly(ADP-ribose) polymerase in tumors from BRCA mutation carriers. $N$ Engl J Med. 2009;361(2):123-134.

49. Russo AL, Kwon HC, Burgan WE, et al. In vitro and in vivo radiosensitization of glioblastoma cells by the poly (ADP-ribose) polymerase inhibitor E7016. Clin Cancer Res. 2009;15(2):607-612.

50. Tsukada Y, Fouad A, Pickren JW, Lane WW. Central nervous system metastasis from breast carcinoma. Autopsy study. Cancer. 1983;52(12):2349-2354.

51. Steeg PS, Camphausen KA, Smith QR. Brain metastases as preventive and therapeutic targets. Nat Rev Cancer. 2011;11(5):352-363.

52. Kesari S, Batchelor TT. Leptomeningeal metastases. Neurol Clin. 2003;21(1):25-66.

53. Herrlinger U, Forschler H, Kuker W, et al. Leptomeningeal metastasis: survival and prognostic factors in 155 patients. J Neurol Sci. 2004; 223(2):167-178.

54. Akeson P, Larsson EM, Kristoffersen DT, Jonsson E, Holtas S. Brain metastases - comparison of gadodiamide injection-enhanced MR imaging at standard and high dose, contrast-enhanced CT and noncontrast-enhanced MR imaging. Acta Radiol. 1995;36(3):300-306.

55. Sze G, Milano E, Johnson C, Heier L. Detection of brain metastases: comparison of contrast-enhanced MR with unenhanced MR and enhanced CT. AJNR Am J Neuroradiol. 1990;11(4):785-791.

56. Nguyen DX, Massague J. Genetic determinants of cancer metastasis. Nat Rev Genet. 2007;8(5):341-352.

57. Frank SA. Genetic predisposition to cancer - insights from population genetics. Nat Rev Genet. 2004;5(10):764-772.

58. Slamon DJ, Clark GM, Wong SG, Levin WJ, Ullrich A, McGuire WL. Human breast cancer: correlation of relapse and survival with amplification of the HER-2/neu oncogene. Science. 1987;235(4785): 177-182.

59. Slamon DJ, Godolphin W, Jones LA, et al. Studies of the HER-2/ neu proto-oncogene in human breast and ovarian cancer. Science. 1989;244(4905):707-712.

60. Miller SJ, Xing X, Xi L, Hung MC. Identification of a specific DNA region required for enhanced transcription of HER2/neu in the MDA-MB453 breast cancer cell line. DNA Cell Biol. 1996;15(9): 749-757.

61. Fuchs IB, Loebbecke M, Buhler H, et al. HER2 in brain metastases: issues of concordance, survival, and treatment. J Clin Oncol. 2002; 20(19):4130-4133.

62. Abbott NJ, Ronnback L, Hansson E. Astrocyte-endothelial interactions at the blood-brain barrier. Nat Rev Neurosci. 2006;7(1):41-53.

63. Igarashi Y, Utsumi H, Chiba $H$, et al. Glial cell line-derived neurotrophic factor induces barrier function of endothelial cells forming the blood-brain barrier. Biochem Biophys Res Commun. 1999; 261(1):108-112.

64. Lee SW, Kim WJ, Choi YK, et al. SSeCKS regulates angiogenesis and tight junction formation in blood-brain barrier. Nat Med. 2003; 9(7):900-906.

65. Fidler IJ, Yano S, Zhang RD, Fujimaki T, Bucana CD. The seed and soil hypothesis: vascularisation and brain metastases. Lancet Oncol. 2002;3(1):53-57.

66. Lin Q, Balasubramanian K, Fan D, et al. Reactive astrocytes protect melanoma cells from chemotherapy by sequestering intracellular calcium through gap junction communication channels. Neoplasia. 2010;12(9):748-754. 
67. Rowley HA, Scialfa G, Gao PY, et al. Contrast-enhanced MR imaging of brain lesions: a large-scale intraindividual crossover comparison of gadobenate dimeglumine versus gadodiamide. AJNR Am JNeuroradiol. 2008;29(9):1684-1691.

68. Nguyen DX, Bos PD, Massague J. Metastasis: from dissemination to organ-specific colonization. Nat Rev Cancer. 2009;9(4):274-284.

69. Weil RJ, Palmieri DC, Bronder JL, Stark AM, Steeg PS. Breast cancer metastasis to the central nervous system. Am J Pathol. 2005;167(4): 913-920.

70. Fitzgerald DP, Palmieri D, Hua E, et al. Reactive glia are recruited by highly proliferative brain metastases of breast cancer and promote tumor cell colonization. Clin Exp Metastasis. 2008;25(7):799-810.

71. Loscher W, Potschka H. Drug resistance in brain diseases and the role of drug efflux transporters. Nat Rev Neurosci. 2005;6(8):591-602.

72. Eisenblatter T, Huwel S, Galla HJ. Characterisation of the brain multidrug resistance protein (BMDP/ABCG2/BCRP) expressed at the blood-brain barrier. Brain Res. 2003;971(2):221-231.

73. Breedveld P, Pluim D, Cipriani G, et al. The effect of Bcrp1 (Abcg2) on the in vivo pharmacokinetics and brain penetration of imatinib mesylate (Gleevec): implications for the use of breast cancer resistance protein and P-glycoprotein inhibitors to enable the brain penetration of imatinib in patients. Cancer Res. 2005;65(7):2577-2582.

74. Cisternino S, Mercier C, Bourasset F, Roux F, Scherrmann JM. Expression, up-regulation, and transport activity of the multidrugresistance protein Abcg2 at the mouse blood-brain barrier. Cancer Res. 2004;64(9):3296-3301.

75. Regina A, Demeule M, Laplante A, et al. Multidrug resistance in brain tumors: roles of the blood-brain barrier. Cancer Metastasis Rev. 2001;20(1-2):13-25.

76. Becker I, Becker KF, Meyermann R, Hollt V. The multidrug-resistance gene MDR1 is expressed in human glial tumors. Acta Neuropathol. 1991;82(6):516-519.

77. Toth K, Vaughan MM, Peress NS, Slocum HK, Rustum YM. MDR1 P-glycoprotein is expressed by endothelial cells of newly formed capillaries in human gliomas but is not expressed in the neovasculature of other primary tumors. Am J Pathol. 1996;149(3):853-858.

78. Haga S, Hinoshita E, Ikezaki K, et al. Involvement of the multidrug resistance protein 3 in drug sensitivity and its expression in human glioma. Jpn J Cancer Res. 2001;92(2):211-219.

79. Graff JR, Gabrielson E, Fujii H, Baylin SB, Herman JG. Methylation patterns of the E-cadherin $5^{\prime} \mathrm{CpG}$ island are unstable and reflect the dynamic, heterogeneous loss of E-cadherin expression during metastatic progression. J Biol Chem. 2000;275(4):2727-2732.
80. Nass SJ, Herman JG, Gabrielson E, et al. Aberrant methylation of the estrogen receptor and E-cadherin 5' $\mathrm{CpG}$ islands increases with malignant progression in human breast cancer. Cancer Res. 2000; 60(16):4346-4348.

81. Deli MA, Descamps L, Dehouck MP, et al. Exposure of tumor necrosis factor-alpha to luminal membrane of bovine brain capillary endothelial cells cocultured with astrocytes induces a delayed increase of permeability and cytoplasmic stress fiber formation of actin. JNeurosci Res. 1995;41(6):717-726.

82. Didier N, Romero IA, Creminon C, Wijkhuisen A, Grassi J, Mabondzo A. Secretion of interleukin-1 beta by astrocytes mediates endothelin-1 and tumour necrosis factor-alpha effects on human brain microvascular endothelial cell permeability. J Neurochem. 2003;86(1):246-254.

83. Emerich DF, Snodgrass P, Dean R, et al. Enhanced delivery of carboplatin into brain tumours with intravenous Cereport (RMP-7): dramatic differences and insight gained from dosing parameters. Br J Cancer. 1999;80(7):964-970.

84. Huwyler J, Wu D, Pardridge WM. Brain drug delivery of small molecules using immunoliposomes. Proc Natl Acad Sci U S A. 1996; 93(24):14164-14169.

85. Thiery JP. Epithelial-mesenchymal transitions in tumour progression. Nat Rev Cancer. 2002;2(6):442-454.

86. Li X, Lewis MT, Huang J, et al. Intrinsic resistance of tumorigenic breast cancer cells to chemotherapy. J Natl Cancer Inst. 2008;100(9) 672-679.

87. Husemann Y, Geigl JB, Schubert F, et al. Systemic spread is an early step in breast cancer. Cancer Cell. 2008;13(1):58-68.

88. Norton L, Massague J. Is cancer a disease of self-seeding? Nat Med. 2006;12(8):875-878.

89. Joyce JA, Pollard JW. Microenvironmental regulation of metastasis. Nat Rev Cancer. 2009;9(4):239-252.

90. Hurwitz H, Fehrenbacher L, Novotny W, et al. Bevacizumab plus irinotecan, fluorouracil, and leucovorin for metastatic colorectal cancer N Engl J Med. 2004;350(23):2335-2342.

91. Paez-Ribes M, Allen E, Hudock J, et al. Antiangiogenic therapy elicits malignant progression of tumors to increased local invasion and distant metastasis. Cancer Cell. 2009;15(3):220-231.

92. Seton-Rogers S. When good drugs do bad things. Nat Rev Cancer. 2009;9:228-229.
Breast Cancer: Targets and Therapy

\section{Publish your work in this journal}

Breast Cancer: Targets and Therapy is an international, peerreviewed open access journal focusing on breast cancer research, identification of therapeutic targets and the optimal use of preventative and integrated treatment interventions to achieve improved outcomes, enhanced survival and quality of life for the cancer patient.

\section{Dovepress}

View the full aims and scopes of this journal here. The manuscript management system is completely online and includes a very quick and fair peer-review system, which is all easy to use. Visit http:// www.dovepress.com/testimonials.php to read real quotes from published authors. 